\title{
microRNAs as therapeutic targets in intestinal diseases
}

\author{
Chen Wang ${ }^{1}$ and Jiangning Chen ${ }^{1,2^{*}}$
}

\begin{abstract}
Intestinal diseases, which primarily include inflammatory bowel disease (IBD), colorectal cancer (CRC) and colitis associated cancer (CAC), are believed to develop as a result of heredity transmit, autoimmune disorders, environmental factors and intestinal flora dysbiosis. As small non-coding RNAs, microRNAs (miRNAs) are vital bioactive factors that are important at almost each kind of cellular events. Recent studies have led to novel perceptivity into the roles of miRNAs in the pathogenetic process of many diseases, including intestinal diseases. Thus, miRNAs represent potential goldmines for basic research and therapeutic applications. The present review outlines the research advances of miRNAs in IBD, CRC and CAC, with an emphasis on their application values as therapeutic targets and the associated challenges.
\end{abstract}

Keywords: microRNAs, Inflammatory bowel disease, Colorectal cancer, Therapeutic targets

\section{Background}

Recently, the incidence of intestinal diseases, including inflammatory bowel disease (IBD), colorectal cancer (CRC) and colitis associated cancer (CAC), has been boosted significantly. However, existing medical approaches are unable to meet the requirements of eliminating these diseases. Unravelling the pathogenic mechanism of intestinal inflammation and cancer may favor the development of novel diagnostic and treatment methods.

IBD, mainly including Crohn's disease (CD) and ulcerative colitis (UC), is a chronic intestinal immune disorder with characteristics that develop through a complicated combination of inheritance, inflammatory responses and environmental triggers [1]. CD may attack any site of the gastrointestinal tract, nevertheless UC typically happens in the colon. Although extensively studied over the past 20 years, the origination and development of IBD remain unknown to a great extent [2]. Until recently, researches have concentrated on small non-coding RNAs (ncRNAs) associated with inflammation to uncover the molecular manners in IBD. With respect to these ncRNAs, precise regulatory relationships are embodied in extensive gene expression alterations that exist in both CD and UC [3].

\footnotetext{
* Correspondence: jnchen@nju.edu.cn

${ }^{1}$ State Key Laboratory of Pharmaceutical Biotechnology, School of Life Sciences, Nanjing University, Nanjing 210023, People's Republic of China ${ }^{2}$ State Key Laboratory of Analytical Chemistry for Life Sciences and Collaborative Innovation Center of Chemistry for Life Sciences, Nanjing University, Nanjing 210023, People's Republic of China
}

CRC serves as one of the most common cancers globally, and liver metastasis ranks as the major cause of CRC associated deaths. Although the pathogenesis of CRC is not fully understood, more and more studies are seeking epigenetic patterns of CRC, with a notable progress in understanding the importance of DNA methylation, histone modifications and ncRNAs. ncRNAs contain a variety of RNA transcripts that cannot express into proteins but rather influence the target genes at transcriptional or post-transcriptional levels $[4,5]$. Among the known types of ncRNAs, the most studied is miRNA, research on which with respect to intestinal diseases may aid our acknowledgement of their development and accelerate clinical progress $[6,7]$.

\section{Main text miRNA overview}

In 1993, Lee et al. discovered the first miRNA, Lin-4 small RNA, which make a difference to the growth of Caenorhabditis elegans [8]. miRNAs are single-stranded RNAs, having a length from 19 to 24 nucleotides.

Firstly transcribed as primary molecules from the genome, miRNAs are then transformed into precursor and mature forms with the help of proteins such as Drosha and Dicer. miRNAs exert biological functions by complementary pairing within the 3 '-untranslated region (3'-UTR) of specific mRNAs, which typically leads to a downregulation of target gene expression by the way of 
mRNA degradation or translational inhibition [9, 10]. Approximately three quarters of the human genome are transcribed into RNA, of which just $3 \%$ is transcribed into mRNAs, which can encode proteins [11]. Thus, the number of ncRNAs is much larger than that of RNAs with protein-coding potentials. Moreover, it has be predicted that miRNAs may hold the promise to adjust almost a third of mammal genes.

Since the discovery of miRNAs involved in regulating the expression of an oncogene or tumour suppressor ten years ago $[12,13]$, the characteristics of miRNAs in various human diseases has been widely studied [14]. The regulatory activity of miRNAs typically involves the translational repression of target mRNAs or the decrease of mRNA stability, resulting in a reduction of the final protein output from a given mRNA transcript. Thus, the abnormal expression of miRNAs often results in aberrant cellular functions, leading to further changes in downstream gene regulation and signalling cascades. With the development of miRNA arrays and RNA high-throughput sequencing techniques, miRNA dysregulation has been observed in tissue samples from patients with intestinal diseases $[15,16]$. Based on identified physiological and pathological clues, miRNAs may exert a significant impact on intestinal diseases. Progress in the determination and research of miRNAs demonstrated their specific levels and functional manners as pro-/anti-inflammatory genes, oncogenes or tumour suppressors. Thus, in-depth analyses of miRNA-associated functional biomolecules in specific cell systems and different animal models may help to illuminate the roles of miRNAs in the pathogenic processes.

As the dysregulation and function of miRNAs in intestinal diseases have become better understood, the development of miRNA-based therapeutics has rapidly advanced. Here, we summarize recent insights into the opportunities and challenges associated with investigating therapeutic targets of miRNAs in intestinal disease and discuss how these functional molecules can be used for new drug development.

\section{miRNAs as therapeutic targets in CD}

Recent research has analysed the miRNA expression profiles in tissues from $\mathrm{CD}$ patients. The results of miRNA array and real-time polymerase chain reaction (RT-PCR) demonstrated that miR-21, miR-23b, miR-106a, miR-191, miR-223, miR-594 and miR-16 were significantly upregulated in inflamed sites compared to normal sites. Whereas, miR-19b and miR-629 were underexpressed $[17,18]$. Of these miRNAs, inverse relationships between miR-106a and IL-10, miR-150 and c-Myb were observed. These findings may provide new understandings into the regulatory mechanism of gastrointestinal disorders and demonstrate the potential of therapeutic miRNAs. Theoretically, recovering
decreased-miRNAs by means of miRNA mimics, or inhibiting upregulated-miRNAs using specific inhibitors may offer clinical application of altered physiological processes in $\mathrm{CD}$. Unlike siRNAs usually with a unique molecule target, miRNAs typically regulate various genes and signallings, and therefore have a comprehensive therapeutic effect.

With respect to restoring miRNA activity, two primary ways can be accomplished, which contain the delivery of chemosynthetic mimics, plasmid vectors or viral vectors [19]. Especially regarding IBD treatments, underlying therapeutic ways of manipulating miRNAs comprise blocking the progression of inflammation or improving the sensitivity of cells to conventional therapies. As mentioned above, the treatment approaches of miRNA-associated intervention contain miRNA inhibitors and miRNA mimics. As one type of miRNA inhibitors, antagomirs can bind to and inhibit the expression of a miRNA of interest, further rescue the inhibition of its targets. In contrast, miRNA mimics are applied to recover the changed function through the restoration of miRNAs into pathological cells to regain the physical state [20].

Several studies have highlighted the possible therapeutic applications of upregulated miRNAs, which involve miR155 and miR-31. Served as one of the most well investigated miRNAs, miR-155 exerts a major regulatory function in inherent and acquired immunity and is upregulated in active UC and CD tissues. One target gene of miR-155 is inhibitor of cytokine signalling (SOCS-1), which is a subtractive manipulator of the lipopolysaccharide-caused activation of macrophages as well as the antigen prebeatification of dendritic cells. More targets of miR-155 contain Janus kinase and signal transducer activator of transcription, which belong to the JAK/STAT signalling pathway [21]. In addition, IL-10-regulated miR-155 plays a crucial role in commensal bacterial-induced colitis by targeting SH2-containing inositol phosphatase-1 (SHIP-1). Inhibitors against miR-155 could lead to the recovery of SOCS-1 and SHIP-1 expression and the activation of the immune system, thereby alleviating intestinal inflammation $[22,23]$. Brest et al. [24] found that the expression of miR-196 is increased in colon epithelial cells exhibiting CD-associated inflammation in contrast to control samples. miR-196 was observed to downregulate the protective immunity-related GTPase family M protein (IRGM), which affected the efficacy of autophagy, thereby implicating it as an intervention target. Overexpression of miR-124 promoted gut immunity by regulating aryl hydrocarbon receptors in $\mathrm{CD}$, and intestinal delivery of miR-124 inhibitors ameliorated trinitrobenzene sulfonic acid (TNBS)-triggered colitis [25]. Wang et al. [26] showed that miR-223 is overexpressed in IBD and functions as an important mediator in the crosstalk between Claudin-8 (CLDN8) and the IL23 signal pathway. Treatment with miR-223 inhibitors could upregulate CLDN8 and ameliorate TNBS-induced colitis. 
Nguyen et al. [27] revealed that miR-30c and miR-130a were overexpressed in CD and may directly target the 3'-UTRs of ATG5 and ATG16L1, respectively. Furthermore, this group demonstrated that suppressors of miR-30c and miR-130a inhibited AIEC-induced downregulation of ATG5 and ATG16L1 expression, enhanced autophagy capacity and decreased inflammation in vivo. Elevated miR-301a could negatively regulate SMAD nuclear interacting protein 1 (SNIP1) and stimulate Th17 cell differentiation through direct regulation of SMAD nuclear interacting protein 1 (SNIP1). Colonic delivery of miR-301a inhibitors downregulated the levels of inflammatory molecules in the inflamed sites caused by TNBS [28]. Additionally, Shi et al. [29] demonstrated that the miR-31 level inversely correlated with that of IL-25 in the large intestine of colitis mice and CD patients. Altering colonic miR-31 levels could affect the mucosal Th1/Th17 pathway adjusted by IL-12/23 and influence the intestinal immunity. Recovering the level of IL-25 and inhibiting Th1/Th17 immunoreactivity through intestinal delivery of miR-31 inhibitors could effectively alleviate the colitis in mice.

With respect to the downregulated miRNAs, Nguyen et al. $[27,30]$ examined tissue samples from $8 \mathrm{CD}$ patients and observed that the expression of miR-7 was decreased compared to 6 healthy persons. They revealed that miR-7 was decreased in colon sites associated with CD inflammation, whereas its target, CD98, was upregulated, showing the potential of this molecule for future therapeutic interventions. In artificially induced colitis mice, miR-210 suppressed the expression of hypoxia-inducible factor $1 \alpha(H I F 1 \alpha)$, a key transcription factor of Th17 polarisation, which further promoted a negative feedback circuit mediated by HIF1 $\alpha$ and controlled disease severity [31]. Recently, Huang et al. [32] demonstrated that miR-141 exert a crucial function in colonic leucocyte trafficking by targeting CXCL12 $\beta$. The interaction between miR-141 and the mRNA of CXCL12 $\beta$ effects leukocyte trafficking and the recruitment of immune cells, further regulating the intestinal inflammation in the development of CD. Inhibitors of miR-141 were observed to aggravate TNBS-induced colitis through suppressing CXCL12 $\beta$. In contrast, treatment with miR-141 protected mice against the pathogenesis of TNBScaused and IL-10 knockout-induced spontaneous enteritis. The characteristics of miRNAs with therapeutic potential in CD patients were listed in Table 1 . These findings mentioned above have prominently deepened our understanding with respect to the development of $\mathrm{CD}$ and have showed the clinical application of miRNAs as intervention targets for $\mathrm{CD}$ treatment.

\section{miRNAs as therapeutic targets in UC}

In 2008, Wu et al. [17] established the first differential expression profile of miRNAs in IBD patients. They detected RNA samples extracted from colon tissues of UC patients compared with healthy control subjects. The results revealed that miR-23a, miR-16, miR-21, miR-29a, miR-24, miR-195, let-7f and miR-126 were prominently overexpressed in tissues from UC patients compared to healthy persons. Whereas, miR-375, miR-192 and miR-422b were markedly underexpressed. Other studies confirmed that the levels of miR-155, miR-127-3p, miR-324-3p, miR-127-3p, miR-31, miR-7, miR-223, miR-324-3p, miR-150 and miR-20b were increased in inflammatory sites of colon tissues from UC patients compared to control sites [18, 34, 33, 35-38], whereas miR-346, miR-215, miR-320a, miR-200b, let-7, miR-188-5p, miR-125, miR-101, miR-25 and miR-26 were downregulated [18, 39, 40, 41].

Among these miRNAs, miR-192 was observed to be the most significantly overexpressed miRNA in tissue samples from UC patients. Macrophage inflammatory peptide- $2 \alpha$ (MIP- $2 \alpha)$ was shown be to negatively regulated by miR-192 in intestinal epithelial cells (IECs), which functions as a CXC chemokine [17]. Another research demonstrated that TGF $\beta$ can trigger miR-192 expression [42], suggesting that miR-192 could be a primary modulator of the inflammation response. Furthermore, it has been found that miR-126 was overexpressed in UC patient tissues, and it was confirmed that this miRNA could target ІкB $\alpha$, a suppressor of the NF- $\kappa B$ signalling [36]. Given the decreased expression and function of miR-126 in active UC, miR-126 mimics may hold therapeutic potential for UC treatment [43]. As a vital regulator in the inflammatory response, miR-155 exerts important functions in both CD and UC. miR-155 has been shown to have an important role between the Th1 and Th2 pathways by repressing SOCS-1, which performs a protective function under the situation of infections but is pernicious in inflammatory bowel disease [44]. Recently, it was revealed that miR-155 was upregulated in patients with severe UC [33]. Furthermore, miR-155 knockout mice were observed to be resistant to the intestinal inflammation caused by dextran sulfate sodium (DSS) compared to control mice [45]. Additionally, it has been reported that miR-150 is overexpressed in colon tissues from $\mathrm{UC}$ and $\mathrm{CD}$ patients and in mice with intestinal inflammation. The upregulation of miR-150 may indicate enhanced leukocyte infiltration in the mucosa, leading to a diseased state [37]. Compared to control tissue samples, miR-124 was found to decrease in the colon tissue samples from UC patients. miR-124 effects the protein levels of signal transducer and activator of transcription 3 (STAT3), and the downexpression of this miRNA could result in the overexpression of STAT3 and the enhancement of inflammation [40]. In addition, overexpression of miRNA-214 could suppress the protein levels of phosphatase and tensin homologue (PTEN) and PDZ and LIM domain 2 (PDLIM2), induce raised phosphorylation level of NF- $\mathrm{kB}$ and subsequent IL-6 secretion [46]. Furthermore, 
Table 1 miRNA characteristics in CD patients

\begin{tabular}{|c|c|c|c|c|}
\hline miRNA & Target gene(s) & Dysregulation & Function & Reference \\
\hline miR-155 & SOCS-1, JAK/STAT, SHIP-1 & up & $\begin{array}{l}\text { A negative regulator of lipopolysaccharide-induced macrophage activation } \\
\text { and antigen presentation by dendritic cells }\end{array}$ & {$[21-23]$} \\
\hline miR-196 & IRGM & up & Affects autophagy efficacy & [24] \\
\hline miR-124 & Aryl hydrocarbon receptor & up & Promotes intestinal inflammation & [25] \\
\hline $\operatorname{miR}-233$ & CLDN8 & up & A mediator of the crosstalk between the CLDN8 and IL23 signal pathways & [26] \\
\hline miR-30c & ATG5 & up & Decreases autophagic activity and increases inflammation & [27] \\
\hline miR-130a & ATG16L1 & up & Decrease autophagic activity and increases inflammation & [27] \\
\hline miR-301a & $\begin{array}{l}\text { SMAD Nuclear Interacting } \\
\text { Protein } 1\end{array}$ & up & Promotes Th17 cell differentiation & [28] \\
\hline miR-31 & IL25 & up & Affects the mucosal IL-12/23-mediated Th1/Th17 pathway & {$[29]$} \\
\hline miR-7 & CD98 & down & Provides a target for future therapeutic interventions & {$[30]$} \\
\hline miR-210 & HIF1a & down & A key transcription factor of Th17 polarisation & [31] \\
\hline miR-141 & CXCL12 $\beta$ & down & Influences immune cell recruitment, alters leukocyte trafficking & [32] \\
\hline
\end{tabular}

miR-214 inhibitors can markedly inhibit the phosphorylation of NF- $\mathrm{kB}$, alleviate colitis both in experimental mice caused by DSS and in UC patients. Whereas, the intraperitoneal administration of miR-665 inhibitors significantly aggravate the intestinal inflammation in DSS-caused colitis, which appears to be with shorter colon length and hematochezia [47]. Alicaforsen is an example of an mRNA targeting oligonucleotide therapy that inhibits the protein production of the intercellular cell adhesion molecule-1 (ICAM-1) in the development of UC [48]. Thus, inhibitors of miR-155, miR-150 and miR-124 were currently demonstrated to hold promise as candidates for the clinical application of inflammatory bowel disease. The characteristics of miRNAs with therapeutic potential in UC patients were listed in Table 2. Based on the findings above, by affecting various immune responses, miRNAs and their inhibitors would be very promising for the clinical application of the inflammation-associated diseases in the intestines.

\section{miRNAs as therapeutic targets in intestinal cancer}

In addition to IBD, miRNAs simultaneously play a crucial part in intestinal cancer. Increased levels of miR-21 in tissues were observed to be relevant to a low survival rate, distant metastatic development and positive lymph nodes of CRC patients [13]. Cellular miR-21 increases the metastasis of CRC cells [49] and was also shown to reduce 5-FU-caused G2/M phases progression and cell apoptosis by inhibiting the protein expression of mutS homolog 2 (MSH2) [50, 51]. Therefore, miR-21 may emerge as a promising biomarker for the prognosis of CRC patients and also would be an intervention target for CRC treatment. Sun et al. [52] demonstrated that miR-31 was the most significantly dysregulated miRNA in CRC patient tissues using miRNA arrays. miR-31 promotes the activation of the RAS signalling pathway, stimulating CRC cell growth and tumourigenesis through negatively regulating Ras p21 protein activator 1 (RASA1). Furthermore, they analysed other miRNAs that probably regulating RASA1 in CRC. Upregulation of miR-223 was detected and shown to be involved in the downregulation of RASA1 in tissues from CRC patients. The results of xenograft mice model showed that the overexpression of miR-223 can stimulate tumour growth in vivo, whereas miR-223 inhibitors may suppress the tumour growth established with CRC cells [53]. Additional identified targets of miR-31 include FIH-1 and T cell lymphoma invasion and

Table 2 miRNA characteristics in UC patients

\begin{tabular}{lllll}
\hline miRNA & Target gene(s) & Dysregulation & Function & Reference \\
\hline miR-192 & MIP-2a & up & A master regulator of the inflammation process & [42] \\
miR-126 & IkBa & up & Inhibits the NF-kB signalling pathway & [43] \\
miR-155 & SOCS-1 & up & Protective in the context of infections, but pernicious in autoimmune disease & {$[44,45]$} \\
miR-150 & c-Myb & up & Increases mucosal leukocyte infiltration & [33] \\
miR-124 & STAT3 & down & Inhibits STAT3 activation & [40] \\
miR-214 & PDLIM2, PTEN & up & Induces NF-KB phosphorylation and subsequent IL-6 secretion & [46] \\
miR-665 & XBP1, ORMDL3 & up & Promotes apoptosis and colitis & [47] \\
\hline
\end{tabular}


metastasis 1 (TIAM1), which control cell proliferation and metastasis in CRC. Moreover, treatment using miR-31 inhibitors can enhance the susceptibility of CRC cells to chemotherapeutics, such as 5-FU [54]. Because miR-31 and miR-223 may synergistically function in CRC, the combinational use of inhibitors for miR-31 and miR-223 would result in a superior suppression of RASA1. Additionally, miR-143 was detected to be markedly downregulated in CRC tissues, and the upregulation of miR-143 led to a decrease in cell proliferation, increased cell death and enhanced chemosensitivity to 5-FU [55]. Belong to one cluster, the tumour suppressor miR-145 was demonstrated to inhibit tumour growth and metastasis in CRC $[56,57]$. In general, miR-143 and miR-145 share target genes in CRC, which includes mouse double minute 2 homolog (MDM2), erb-b2 receptor tyrosine kinase 3 (ERBB3), Kruppel like factor 5 (KLF5), BRAF and CD44. The simultaneous overexpression of miR-143 and miR-145 was indicated to potentially have a synergistic anti-tumour effect $[58,59]$.

In addition, several important miRNAs were identified as having roles in CRC proliferation and metastasis. The level of miR-137 was shown to negatively correlated with that of cell division cycle 42 (CDC42), which is a member of the Rho GTPase family and plays an impact in CRC initiation and progression [60]. Furthermore, the expressions of miR-34a and miR-199a were both observed to be negatively correlated with that of $\mathrm{Axl}$, which is a receptor tyrosine kinase that induces the growth and metastasis of cancer cells. Overexpression of miR-34a or miR-199a inhibited cell metastasis and decreased the number of liver metastatic nodules in vivo [61]. Hansen et al. [62] demonstrated that the high level of miR-126 promotes cell proliferation and metastasis through negatively regulating insulin receptor substrate 1 (IRS-1) and AKT and ERK1/2 signalling pathways in CRC. Because vascular endothelial growth factor A (VEGF-A) is also a regulatory molecule of miR-126, manipulating miR-126 expression may simultaneously provide anti-proliferation, anti-metastasis and anti-angiogenic therapy effects [63].

Furthermore, some miRNAs are involved in the resistance to the chemotherapy using for CRC. As a regulatory target of miR-34a, SIRT-1 is related to the resistance of cells to drugs. Akao et al. observed that miR-34a upregulation can effectively improve the 5-FU resistance of CRC cells [64]. With respect to the chemotherapeutics used for metastatic CRC patients, miR-203 was demonstrated to reverse chemoresistance caused by oxaliplatin through targeting ATM kinase and AKT [65, 66]. As a new modulator of BCL-2, miR-129 can promote cell apoptosis, inhibit cell growth and cause cell-cycle progression in CRC. Karaayvaz et al. revealed that miR-129 could also target thymidylate synthase (TYMS), a suppressor of the 5-FU, enhancing the susceptibility of CRC cells to chemotherapeutics [67, 68]. Thus, intervention methods on account of miR-129 would contribute to realize a various-targeted anticancer effectiveness. Wang et al. [69] demonstrated that delivery of let-7 mimics could reduce tumour growth in vivo. Similarly, intravenous injection of miR-502 precursor every third day was shown to inhibit ectopic tumour growth in mice. By negatively regulating NIRF in vitro, the heterotopic replenishment of miR-502 suppressed cell proliferation, cell cycle drive and autophagy of CRC cells. Thus, as a potential tumour suppressor, miR-502 may be a new candidate for developing a CRC treatment strategy [70]. Importantly, according to the characteristics of different miRNAs and the disease stages of CRC patients, the appropriate combination of miRNAs should be considered when making treatment strategies.

Chronic and prolonged inflammation plays a vital role in promoting $\mathrm{CAC}$ tumourigenesis and progression, but the underlying biological events associated with this malignant transformation remain largely unknown. Among the existing findings, several important miRNAs in colitis-associated CRC have been investigated recent years. miR-21 was observed to be significantly upregulated in the tumours from CRC and CAC patients. The inhibition of miR-21 reduced the levels of proinflammatory and procarcinogenic cytokines, while STAT3 and NF-kB inactivation led to the decreased levels of Ki67 and step-down cell growth [71]. The overexpression of miR-26b in tissues was demonstrated to be closely related to the disease severity of $\mathrm{UC}$ or CAC patients. By downregulating the expression of the E3 ubiquitin ligase DIP1, the upregulation of miR-26b was shown to be closely related to the stabilization of death-related protein kinase [72]. For the epithelial to mesenchymal transition (EMT) and metastasis process of CRC cells, the activation of IL-6R/STAT3/miR-34a loop was necessary, and it was also relevant to the lymph node and liver metastasis extent of CRC patients. miR-34 could repress the EMT process and was necessary for IL-6-caused process of EMT and cell invasion. It was found that miR-34a, whose expression depends on p53, could inhibit tumour growth by blocking the feedback loop of IL-6R/ STAT3/miR-34a [73]. Additionally, IL-6 could upregulate STAT3-induced transcriptional activation of miR-214 in IECs, which stimulates the levels of phosphorylated-AKT and activated-NF- $\mathrm{kB}$ by targeting PDLIM2 and PTEN. The active condition of this regulatory loop was closely associated with the degree of UC patients and the malignant transformation to CRC [46]. The level of miR-301a was upregulated in IECs of IBD patients and CAC patients compared with healthy controls. The mice, whose miR-301a was knockout, had an increased epithelial barrier integrity. There appears to be less severe colitis caused by DSS and smaller amounts of tumours induced by azoxymethane administration in these mice than wild type mice 
[74]. Recently, enhanced miR-18a expression was observed to lead to highly activated NF- $\mathrm{kB}$ and STAT3 by negatively regulating the expression of protein inhibitor of activated STAT3 (PIAS3). The feedback loops mediated by miR-18a significantly promoted cell proliferation and inhibited cell apoptosis, thereby driving CAC progression [75]. The characteristics of miRNAs with therapeutic potential in CRC and CAC patients were listed in Table 3. These observations provide novel proofs for the utilization of miRNA regulation as a key strategy for intestinal cancer treatment.

\section{miRNA delivery approaches in intestinal diseases}

Given the roles of miRNAs in intestinal diseases, it is not surprising that miRNA delivery is a promising area for clinical applications. The different patterns of miRNA expressions in specific types of cells or tissues offer novel possibilities for accurate modulation by miRNA intervention. miRNA delivery systems are being developed to either inhibit the expression of miRNAs or recover the function of miRNAs with the inverse role. Effective methods being explored include those that stimulate miRNAs synthetic process, change epigenetic modification, or use chemosynthetic RNA molecules that mimic miRNAs. Similarly, for approaches that inhibit miRNAs based on nucleotide drugs and chemical molecule are both worthy of attention. Because the effectiveness of single stranded RNA drugs in vivo is less one tenth than in vitro, miRNA mimics are often synthesized and administrated as duplexes $[76,77]$. For synthetic RNA, appropriate modifications by means of chemistry are important to enhance combining capacity, increase stability and improve cell ingestion. To realize an in vivo effective therapy, the delivery of miRNA drugs is usually based on functional carriers or viral vectors. Some common viral vectors, containing lentiviral, adenoviral, retroviral and adeno-associated viral (AAV), have been used for miRNA delivery. Of these vectors, because AAV cannot be typically merged into the genome of host, it is nonpathogenic in mammals and allows for continuously highly expression with highly infection efficiency. Of such systems, reassembled AAV vectors have served as the most potential and have been therapeutically applied to clinical trials. AAV has been observed to effectively acquire anti-tumour effect in haemophilia B treatment and is generally well tolerated [78].

For IBD, treatment based on viral vectors have been tested in mice colitis. Lindsay et al. showed that intravenous and colonic administration of an AAV vector containing IL-10 can significantly reduce intestinal inflammation in acute colitis caused by TNBS and chronic spontaneous colitis induced by IL-10 knockout [79, 80]. Nevertheless, miRNA administration based on viral vectors inevitably has security issues, and it is worth considering the accompanying toxicity and immunogenicity when using this delivery system [81]. Thus, innovative methods to efficiently deliver synthetic miRNAs required further study. With possible implications for IBD therapy, there has been some nanoscale carriers under exploration for small RNA transportation, containing polyethylenimine (PEI), liposome, dendrimers and polylactide-coglycolide (PLGA) particles. Other kinds of materials include silica or gold inorganic materials, exosomes, natural polymers such as atelocollagen, chitosan, protamine et al. [82]. Although nanoparticles have lower efficiency than viral vectors, they hold the potentials to reach specific cells or tissues, with the aid of adjustable ligands of nanoparticles and the specific receptors of different cells or tissues. Similar to miRNAs, Zhang et al. demonstrated that unmodified siRNAs could hardly infiltrate the mucosa structure [83]. On

Table 3 miRNA characteristics in CRC and CAC patients

\begin{tabular}{|c|c|c|c|c|}
\hline miRNA & Target gene(s) & Dysregulation & Function & Reference \\
\hline miR-21 & PDCD4 & up & Invasion, intravasation, metastasis, apoptosis, sensitivity to 5-FU & {$[49-51,71]$} \\
\hline miR-31 & RASA1 & up & Proliferation & {$[52]$} \\
\hline $\operatorname{miR}-223$ & RASA1 & up & Proliferation & {$[53]$} \\
\hline miR-31 & HIF1A, FIH1 & up & Proliferation, metastasis, sensitivity to 5 -FU & {$[54]$} \\
\hline miR-143/145 & MDM2, ERBB3, KLF5, BRAF, CD44 & down & Proliferation, metastasis, death, chemosensitivity to 5-FU & {$[55-59]$} \\
\hline $\operatorname{miR}-137$ & CDC42 & down & Cell cycle and invasion & {$[60]$} \\
\hline $\operatorname{miR}-34 a$ & Axl, SIRT-1 & down & Metastasis, chemosensitivity to 5-FU & {$[61,64,73]$} \\
\hline $\operatorname{miR}-126$ & IRS-1, VEGF-A & down & Proliferation, migration and invasion, anti-angiogenic therapy & {$[62,63]$} \\
\hline miR-203 & ATM kinase and AKT & down & Chemosensitivity to oxaliplatin & {$[65,66]$} \\
\hline $\operatorname{miR}-129$ & BCL2, TYMS & down & Apoptosis, cell growth, cell-cycle, sensitivity to 5-FU & {$[67,68]$} \\
\hline miR-502 & RAB1B, p53 & down & Autophagy, cell proliferation, cell cycle, tumour growth & {$[70]$} \\
\hline miR-26b & E3 ubiquitin ligase DIP1 & up & Cell death & {$[72]$} \\
\hline $\operatorname{miR}-214$ & PDLIM2, PTEN & up & Malignant transformation & {$[46]$} \\
\hline miR-18a & PIAS3 & up & Proliferation, cell apoptosis & {$[75]$} \\
\hline
\end{tabular}


account of this finding, they delivered a TNF $\alpha$ siRNA using liposomes as carrier through colonic administration. This delivery system significantly reduced the mRNA level of TNF $\alpha$ and alleviate the intestinal colitis caused by DSS. Moreover, to raise the efficiency and further decrease the toxic and unwanted effects, delivery strategies which accurate to specific cell type are urgently needed. Zuo et al. [84] investigated the targeted delivery of a novel nano-complex into activated colonic macrophages based on galactosylated low molecular weight chitosan (G-LMWC) and an antisense oligonucleotide (ASO) against TNF $\alpha$. Additionally, specific delivery of miR-16 mimics into colon macrophages negatively regulated the expression of TNF- $\alpha$ and IL-12p40 in mice colitis induced by TNBS. Ultimately, G-LMWC associated ASO against TNF $\alpha$ or miR-16 effectively relieved mucoimmune response and intestinal inflammation, mainly through inhibiting the proinflammatory factors secreted by macrophages [85]. Recently, an orally administrated nucleotide-delivery vehicle was designed using cationic konjac glucomannan, phytagel and an antisense oligonucleotide against TNF- $\alpha$. This vehicle can realize the spontaneous release of nucleotide into colonic macrophages via receptor-mediated phagocytosis and alleviate the symptoms of colitis in mice [86]. Huang et al. and Dai et al. reported that intracolonic delivery of miR-141 or miR193a-3p with PEI as carrier could significantly recover the miRNA expression levels in intestinal epithelial cell. As a result, TNBS/IL-10 knockout-caused or dextran sodium sulfate-induced colitis were respectively ameliorated [32, 87]. Additionally, Zhang et al. demonstrated that nanoparticles derived from edible ginger (GDNPs 2) contain high levels of lipids, proteins, miRNAs and ginger bioactive constituents (6-gingerol and 6-shogaol), which can efficiently be taken up by intestinal epithelial cells and macrophages. Oral administration of GDNPs 2 promoted the proliferation of intestinal epithelial cells, regulated the expression of inflammatory factors, prevented the development of chronic colitis and even colitis associated cancer [88]. The use of the non-viral gene vector to inhibit inflammatory factors secreted by macrophages demonstrates a promising treatment method for CD therapy.

For cancer therapy, methods based on manipulating the synthesis and delivery of miRNAs from exosomes exert an important impact on the tumorigenesis and development. The mechanisms by which extracellular miRNAs are loaded into vesicles are currently being explored. Instances of treatments include siRNA transportation using exosomes to across the mice blood-brain barrier and targeted delivery let-7a to negatively regulate epidermal growth factor receptor (EGFR) with the help of exosomes in RAG knockout mice [89, 90]. Additionally, Yin et al. [91] demonstrated that tumour-derived miR-214 by microvesicles
(MVs) efficiently downregulated PTEN in mouse peripheral $\mathrm{CD}^{+}{ }^{+} \mathrm{T}$ cells, promoting Treg expansion and tumour growth. The intravenous injection of MVs containing miR-214 inhibitors into mice with heterotopic tumours suppressed Treg expansion and tumour progression. Among nucleic acid drugs, miRNAs are promising candidates for endogenous stimuli because of their important functions in tumourigenesis and prominently differential expression. Zhang et al. subtly manufactured nanoscale carriers for the miRNA-modulated delivery of doxorubicin/siRNA, to realize the amplification of miRNAs with low abundance. By modulating the expression of NIR laser, miRNA or ATP, the therapeutic method was assessed respectively in five kinds of tumour cells, resulting in efficient gene silencing and severe apoptosis. The combined utilization of nanophase materials and DNA machines has opened a new avenue for manufacturing nanodrugs for the disease treatment [92]. As for intestinal cancer, intratumoral miRNA delivery using PLGA/PEI-mediated miRNA vector system was recently validated. Liang et al. assembled PLGA/PEI/HA complexes by crosslinking PEI/PLGA suspension with hyaluronic acid, which successfully delivered miRNA plasmid into colon cancer cells and exerted a significant suppressive effect on xenograft tumour in vivo [93]. As a result of administration of siDCAMKL-1, which was encapsulated in Poly (lactide-co-glycolide)-based NPs (NP-siDCAMKL-1), downregulation of oncogenes and reduced tumour growth were achieved [94]. Additionally, several studies specifically delivered miRNA to tumour cells through intravenous injection. Systemic delivery of miR-204-5p carried by PEGylated PLGA nanoparticles or miR-15a/16-1 plasmids encapsulated in cationic liposome respectively caused a significant inhibition of subcutaneous tumour growth in xenograft model of colon cancer [95, 96]. Mesoporous silica nanoparticles have also been used to load miR-328 mimics or miR-155 inhibitors, which resulted in significantly high targeting efficiency and effective therapeutic effects in vitro and in vivo [97, 98]. However, these systems are designed for ectopic tumours, their efficiency for delivering miRNA drugs into intestinal tumour cells requires to be evaluated in the future. Recently, it was reported that intracolonic administration of lentivirus carrying antisense for miR-18a significantly inhibited cell proliferation and led to reduced tumour sizes/numbers in both xenograft colorectal cancer model and AOM-DSS-induced colitis associated colon cancer [75]. Further research concerning in situ drug delivery system at different gut section will lay the foundation for additional clinical study and miRNA-treatment in intestinal diseases.

\section{Challenges of miRNA therapeutics}

In the last two decades, the discovery and research of miRNAs have deppened our understanding of their biological roles. In intestinal diseases, functional alterations 
caused by dysregulated miRNA expression profiles may increase our understanding of disease pathogenesis. As inflammation and cancer are multifactorial diseases, the primary advantage of using miRNA therapeutics is that miRNAs can regulate various genes at transcriptional and post-transcriptional levels, which are associated with one or multiple pathways in the pathological process of intestinal diseases. That is to say, the multi-targeting characteristic of miRNAs offers a possibility to an extensive gene interference by means of limited several miRNAs. Thus, miRNAs gain an advantage over some siRNAs particularly devised to negatively regulate specific target genes [99].

Nevertheless, there exist significant challenges related to therapeutic methods based on miRNAs. As mentioned above, each miRNA may regulate numerous mRNAs [5], and at the same time individual mRNA may be regulated by more than one miRNA, thus creating complex biological regulation. Therefore, predicted miRNAs and their regulated-mRNAs are not limited to the established targeted relationship and specific biological effects. The involvement of other undetermined effects of miRNA treatment should be noted, as they may potentially lead to unwanted consequence. Instances contain germline excision of miR-17-92, which is an oncogenic cluster, finally led to bone development deficiency in vivo [100]. There also exist miRNAs-induced needless effects. One underlying challenge is selectively to deliver miRNAs to the specific organ, because systemic delivery may bring the unexpected consequences. Such as, miR-26a can exert a suppressive function on liver cancer by regulating PTEN, while it plays a tumour promoting role in the development of glioma [101, 102]. On account of the multiple target effect of miRNAs, the side effects including unanticipated off-target effects of miRNAs and potential immunogenicity triggered by delivery system are possibly appear. The requirement for seriously considering the long or short term biological effects of miRNA theraputics should be further emphasized.

Additionally, the expression and function of miRNA may be confined to specific cell context. Because of diverse profiles of miRNAs, one kind of miRNA can have unlike levels in distinct kinds of cells and exert various functions. Thus, alteration of some miRNAs may play a protective role in specific cells, but has a detrimental function in other cells. Accurate transportation of the curative miRNAs into the objective cells may help to decrease the side effects in vivo, which remains another choke point for the application of miRNA treatment. The importance of this point needs be emphasized in future studies [103]. Moreover, the majority of investigations that have studied miRNA profile in intestinal disease are partial studies to some extent. Many studies do not control for anatomic locations, treatment regimens or the inflammatory status of the biopsies, and are non-uniform for the collection methods of patient cohorts and tissue samples. The differences among groups, usually resulting in unlike conclusions, may be attributed to the absence of accordant methods. Thus, studies with larger cohorts to analyse the therapeutic effect of these approaches are required [104].

Furthermore, the obstacle commonly existing in the oligonucleotides utilization should be conquered for miRNA treatment. The miRNAs therapeutics must overcome possible problems associated with oligonucleotide drugs, such as digestion by RNAses in blood, clearance by kidney, obstruction by vascular barrier and low-rate uptake by specific cells $[105,106]$. Nanoscale particles particularly manufactured for the transportation of miRNAs to target cells may be beneficial to realize this process. In addition, some miRNAs with the same sequence are given modified names on account of genomic sites [107]. More in-depth knowledge of the genome location of miRNAs can offer insights into the adjusted mechanism and dysregulated expression during disease development. How the delivery of RNA complexes with a $5 \mathrm{~nm}$ plus diameter reach the target tissue across the vascular endothelium barrier remains a challenge [108]. Further complicating issue is the lately discovered new mechanism of miRNA, which revealed that miRNA could regulate the protein levels through complementary base pairing with the coding regions of target genes. Once the challenges mentioned above are overcome, the potential opportunity of miRNA treatment will be greatly improved.

Although miRNA study are full of challenges and complicacy, therapeutics based on miRNA has currently being examined in clinical trial. As the well-studied miRNA, miR-21 is almost omnipresent overexpressed in many human tumours, such as colorectal cancer $[109,110]$. More interestingly, miR-21 is the specific miRNA that is usually overexpressed in inflammatory sites associated with IBD symptoms. Additionally, NF-kB, which functions as an important transcription factor in the development of IBD and CRC, can contribute to the upregulation of miR-21, suggesting its roles as proinflammatory factor and oncogene $[111,112]$. Therefore, miR-21 can not only serve as a therapeutic target for intestinal diseases but also hold the possible application for clinical treatment. The roles of miRNAs in intestinal diseases, particularly with respect to cell signalling require more in-depth studies. Due to the progress in transportion approaches and RNA chemistries, miRNA treatment for cancer has been developed by several pharmaceutical companies [113].

The therapeutic effects of miRNA treatment are currently evaluated in several clinical trials. Firstly entered clinical development in 2009, "Miravirsen" is an example of miRNA therapeutics for the treatment of hepatitis $\mathrm{C}$ (HCV), particularly inhibiting miR-122 in the liver 
Table 4 miRNA characteristics in clinical trials

\begin{tabular}{|c|c|c|c|c|c|}
\hline Company & Drug name & miRNA & Disease & Trials & Reference \\
\hline Santaris Pharma A/S and Hoffmann-La Roche & Mirvirasen & miR-122 inhibitor & Hepatitis C & Phase II & {$[114]$} \\
\hline Regulus Therapeutics & RG-101 & miR-122 inhibitor & Chronic hepatitis C & Phase II & / \\
\hline miRagen Therapeutics & MRG-106 & miR-155 inhibitor & Cutaneous T cell lymphoma & Phase I & / \\
\hline miRagen Therapeutics & MRG-201 & miR-29 mimic & Scleroderma & Phase I & / \\
\hline Mirna Therapeutics & MRX34 & miR-34 mimic & Multiple solid tumours & Phase I & {$[118,119]$} \\
\hline EnGenelC & MesomiR-1 & miR-16 mimic & Mesothelioma, non-small cell lung cancer & Phase I & [120] \\
\hline Regulus Therapeutics & RG-125 & miR-103/107 inhibitor & Type 2 diabetes and non-alcoholic fatty liver & Phase I & {$[121]$} \\
\hline Regulus Therapeutics & RG-012 & miR-21 inhibitor & Alport syndrome & Phase I & [122] \\
\hline
\end{tabular}

[114-116]. Another miRNA drug against miR-122, RG-101, was conjugated with a N-acetyl-Dgalactosamine and has been evaluated in clinical trials by Regulus Therapeutics [117]. With regard to miRNA therapeutics for cancer treatment, MRX34, a miR-34 mimic, can efficiently ease symptoms of liver cancer by recovering the expression of miR-34a [118]. However, this trial was terminated due to the adverse toxicities caused by immune reactions. Other miRNAs in clinical trials were characterized and listed in Table 4. These miRNA drugs in clinical trials imply the prospect of miRNA therapies in intestinal diseases. The efficient miRNAs in mouse intestinal disease models are especially promising for further clinical trials. If specific miRNA-targeted relationship can be demonstrated, this therapeutic model can offer more ways of transporting miRNA mimics or miRNA inhibitors into the specific organ or cells with lowered side-effects. Thus, there is great promise that the study of aberrantly expressed miRNAs will aid in the development of personalized therapies for patients with intestinal diseases.

In the near future, it is not difficult to foresee that miRNA treatment which holds well-developed effects will ultimately be applied in clinical settings. Whereas, the potential challenges of miRNA treatment such as immunogenicity, off-target effects and easy degradability are required to be overcome in advance. Thus, deeper and broader studies with respect to miRNAs in different cells are urgently needed. Additionally, studies are required to distinguish the regulated genes and their functions to get a better acknowledgement of the biological effects of miRNAs. Collectively, these findings indicate that miRNAs could be targeted therapeutically and served as new drug targets.

\section{Conclusion}

This review highlights miRNAs with applicable probabilities as therapeutic targets and provides an outlook on the challenges of developing miRNA-based therapies. Ever since the first discovery of the abnormal expression of miRNA, research achievements have identified miRNA profiles in patients with intestinal diseases. Despite existing progress, there remains significant hurdles to the realization of effective miRNA therapies. The molecular networks underlying the post-transcriptional regulation exerted by miRNAs still need to be delineated. Further research will absolutely lay a foundation for more effective clinical evaluation and offer more clues for the development of miRNA drugs, supporting miRNAs as potential therapeutics in intestinal diseases.

\begin{abstract}
Abbreviations
3'-UTR: 3'-untranslated region; AAV: Adeno-associated viral; ASO: Antisense oligonucleotide; CAC: Colitis associated cancer; CD: Crohn's disease; CDC42: Cell division cycle 42; CLDN8: Claudin-8; CRC: Colorectal cancer; DSS: Dextran sulfate sodium; EGFR: Epidermal growth factor receptor; EMT: Epithelial to mesenchymal transition; ERBB3: Erb-b2 receptor tyrosine kinase 3; G-LMWC: Galactosylated low molecular weight chitosan; HIF1a: Hypoxia-inducible factor 1a; IBD: inflammatory bowel disease; ICAM1: Intercellular cell adhesion molecule-1; IECs: Intestinal epithelial cells; IRGM: Immunity-related GTPase family M protein; IRS-1: Insulin receptor substrate 1; JAK/STAT: Janus kinase and activator of transcription; KLF5: Kruppel like factor 5; MDM2: Mouse double minute 2 homolog; MIP-2a: Macrophage inflammatory peptide-2a; miRNA: MicroRNA; MSH2: MutS homolog 2; MVs: Microvesicles.; nCRNAs: non-coding RNAs; PDLIM2: PDZ and LIM domain 2; PEI: Polyethylenimine; PIAS3: Protein inhibitor of activated STAT3;

PLGA: Polylactide-coglycolide; PTEN: Phosphatase and tensin homologue; RASA1: Ras p21 protein activator 1; RT-PCR: Real-time polymerase chain reaction; SHIP-1: SH2-containing inositol phosphatase-1; SNIP1: SMAD nuclear interacting protein 1; SOCS-1: Suppressor of cytokine signalling-1; STAT3: Signal transducer and activator of transcription 3; TIAM1: T cell lymphoma invasion and metastasis 1; TNBS: Trinitrobenzene sulfonic acid; TYMS: Thymidylate synthase; UC: Ulcerative colitis; VEGF-A: Vascular endothelial growth factor A
\end{abstract}

\section{Acknowledgements}

Not applicable.

\section{Funding}

This work was supported by the National Natural Science Foundation of China (31870821, 31571458, 31771550, 81673380, 31400671, J1103512, J1210026), the National Key Research and Development Program of China (2017YFC0909700)

Availability of data and materials Not applicable.

Authors' contributions

C.W. performed analysis of literature and wrote the paper, J.C. supervised and modified the manuscript. Both authors approved the final version.

Ethics approval and consent to participate Not applicable. 


\section{Consent for publication}

Not applicable.

\section{Competing interests}

The authors declare that they have no competing interests.

\section{Publisher's Note}

Springer Nature remains neutral with regard to jurisdictional claims in published maps and institutional affiliations.

\section{Received: 16 August 2018 Accepted: 15 March 2019} Published online: 03 September 2019

\section{References}

1. Xavier RJ, Podolsky DK. Unravelling the pathogenesis of inflammatory bowel disease. Nature. 2007;448(7152):427-34.

2. Sartor RB. Mechanisms of disease: pathogenesis of Crohn's disease and ulcerative colitis. Nat Clin Pract Gastroenterol Hepatol. 2006;3(7):390-407.

3. Khor B, Gardet A, Xavier RJ. Genetics and pathogenesis of inflammatory bowel disease. Nature. 2011:474(7351):307-17.

4. Mattick JS. The genetic signatures of noncoding RNAs. PLoS Genet. 2009;5(4):e1000459.

5. Bartel DP. MicroRNAs: target recognition and regulatory functions. Cell. 2009:136(2):215-33.

6. van Kouwenhove M, Kedde M, Agami R. MicroRNA regulation by RNAbinding proteins and its implications for cancer. Nat Rev Cancer. 2011;11(9): 644-56.

7. Wahlestedt C. Targeting long non-coding RNA to therapeutically upregulate gene expression. Nat Rev Drug Discov. 2013;12(6):433-46.

8. Lee RC, Feinbaum RL, Ambros V. The C. Elegans heterochronic gene lin-4 encodes small RNAs with antisense complementarity to lin-14. Cell. 1993; 75(5):843-54.

9. Lee Y, Ahn C, Han J, Choi H, Kim J, Yim J, et al. The nuclear RNase III Drosha initiates microRNA processing. Nature. 2003;425(6956):415-9.

10. Bartel DP. MicroRNAs: genomics, biogenesis, mechanism, and function. Cell. 2004;116(2):281-97.

11. Pennisi E. Genomics. ENCODE project writes eulogy for junk DNA. Science. 2012;337(6099):1159-61.

12. Chen $X$, Guo $X$, Zhang H, Xiang $Y$, Chen J, Yin Y, et al. Role of miR-143 targeting KRAS in colorectal tumorigenesis. Oncogene. 2009;28(10):1385-92.

13. Asangani IA, Rasheed SA, Nikolova DA, Leupold JH, Colburn NH, Post S, et al. MicroRNA-21 (miR-21) post-transcriptionally downregulates tumor suppressor Pdcd4 and stimulates invasion, intravasation and metastasis in colorectal cancer. Oncogene. 2008;27(15):2128-36.

14. Mendell JT, Olson EN. MicroRNAs in stress signaling and human disease. Cell. 2012;148(6):1172-87.

15. Esteller M. Non-coding RNAs in human disease. Nat Rev Genet. 2011;12(12): 861-74.

16. Calin GA, Croce CM. MicroRNA signatures in human cancers. Nat Rev Cancer. 2006;6(11):857-66.

17. Wu F, Zikusoka M, Trindade A, Dassopoulos T, Harris ML, Bayless TM, et al. MicroRNAs are differentially expressed in ulcerative colitis and alter expression of macrophage inflammatory peptide-2 alpha. Gastroenterology. 2008;135(5):1624-35

18. Fasseu M, Tréton X, Guichard C, Pedruzzi E, Cazals-Hatem D, Richard C, et al. Identification of restricted subsets of mature microRNA abnormally expressed in inactive colonic mucosa of patients with inflammatory bowel disease. PLoS One. 2010;5(10):e13160.

19. Sibley CR, Seow $Y$, Wood MJ. Novel RNA-based strategies for therapeutic gene silencing. Mol Ther. 2010;18(3):466-76.

20. Bader AG, Brown D, Winkler M. The promise of microRNA replacement therapy. Cancer Res. 2010;70(18):7027-30.

21. Evel-Kabler K, Song XT, Aldrich M, Huang XF, Chen SY. SOCS1 restricts dendritic cells' ability to break self tolerance and induce antitumor immunity by regulating IL-12 production and signaling. J Clin Invest. 2006;116(1):90-100

22. Singh UP, Murphy AE, Enos RT, Shamran HA, Singh NP, Guan H, et al. miR155 deficiency protects mice from experimental colitis by reducing $T$ helper type 1/type 17 responses. Immunology. 2014;143(3):478-89.
23. Li Y, Tian Y, Zhu W, Gong J, Guo Z, Guo F, et al. IL-10/microRNA-155/SHIP-1 signaling pathway is crucial for commensal bacteria induced spontaneous colitis. Biochem Pharmacol. 2016;116:100-6.

24. Brest $P$, Lapaquette $P$, Souidi $M$, Lebrigand $K$, Cesaro A, Vouret-Craviari V, et al. A synonymous variant in IRGM alters a binding site for miR-196 and causes deregulation of IRGM-dependent xenophagy in Crohn's disease. Nat Genet. 2011;43(3):242-5.

25. Zhao Y, Ma T, Chen W, Chen Y, Li M, Ren L, et al. MicroRNA-124 promotes intestinal inflammation by targeting aryl hydrocarbon receptor in Crohn's disease. J Crohns Colitis. 2016;10(6):703-12.

26. Wang H, Chao K, Ng SC, Bai AH, Yu Q, Yu J, et al. Pro-inflammatory miR-223 mediates the cross-talk between the IL23 pathway and the intestinal barrier in inflammatory bowel disease. Genome Biol. 2016;17:58.

27. Nguyen HT, Dalmasso G, Müller S, Carrière J, Seibold F, Darfeuille-Michaud A. Crohn's disease-associated adherent invasive Escherichia coli modulate levels of microRNAs in intestinal epithelial cells to reduce autophagy. Gastroenterology. 2014;146(2):508-19.

28. He C, Shi Y, Wu R, Sun M, Fang L, Wu W, et al. miR-301a promotes intestinal mucosal inflammation through induction of IL-17A and TNF-a in IBD. Gut. 2016;65(12):1938-50.

29. Shi $T$, Xie $Y, F u Y, Z$ hou $Q, M a Z, M a ~ J$, et al. The signaling axis of microRNA31/interleukin-25 regulates Th1/Th17-mediated inflammation response in colitis. Mucosal Immunol. 2017;10(4):983-95.

30. Nguyen HT, Dalmasso G, Yan Y, Laroui H, Dahan S, Mayer L, et al. MicroRNA7 modulates CD98 expression during intestinal epithelial cell differentiation. J Biol Chem. 2010;285(2):1479-89.

31. Wang $\mathrm{H}$, Flach $\mathrm{H}$, Onizawa M, Wei L, McManus MT, Weiss A. Negative regulation of Hif1a expression and $\mathrm{TH} 17$ differentiation by the hypoxiaregulated microRNA miR-210. Nat Immunol. 2014;15(4):393-401.

32. Huang Z, Shi T, Zhou Q, Shi S, Zhao R, Shi H, et al. miR-141 regulates colonic leukocytic trafficking by targeting CXCL12 $\beta$ during murine colitis and human Crohn's disease. Gut. 2014;63(8):1247-57.

33. Takagi T, Naito Y, Mizushima K, Hirata I, Yagi N, Tomatsuri N, et al. Increased expression of microRNA in the inflamed colonic mucosa of patients with active ulcerative colitis. J Gastroenterol Hepatol. 2010;25(Suppl 1):S129-33.

34. Yang $Y$, Ma $Y$, Shi $C$, Chen $H$, Zhang $H$, Chen $N$, et al. Overexpression of miR-21 in patients with ulcerative colitis impairs intestinal epithelial barrier function through targeting the rho GTPase RhoB. Biochem Biophys Res Commun. 2013:434(4):746-52.

35. Lin J, Welker NC, Zhao Z, Li Y, Zhang J, Reuss SA, et al. Novel specific microRNA biomarkers in idiopathic inflammatory bowel disease unrelated to disease activity. Mod Pathol. 2014;27(4):602-8.

36. Feng X, Wang H, Ye S, Guan J, Tan W, Cheng S, et al. Up-regulation of microRNA-126 may contribute to pathogenesis of ulcerative colitis via regulating NF-kappaB inhibitor IkBa. PLoS One. 2012;7(12):e52782.

37. Bian Z, Li L, Cui J, Zhang H, Liu Y, Zhang CY, et al. Role of miR-150-targeting c-Myb in colonic epithelial disruption during dextran sulphate sodiuminduced murine experimental colitis and human ulcerative colitis. J Pathol. 2011;225(4):544-53.

38. Coskun M, Bjerrum JT, Seidelin JB, Troelsen JT, Olsen J, Nielsen OH. miR-20b, miR-98, miR-125b-1*, and let-7e* as new potential diagnostic biomarkers in ulcerative colitis. World J Gastroenterol. 2013;19(27):4289-99.

39. Chen Y, Xiao Y, Ge W, Zhou K, Wen J, Yan W, et al. miR-200b inhibits TGF$\beta 1$-induced epithelial-mesenchymal transition and promotes growth of intestinal epithelial cells. Cell Death Dis. 2013:4:e541.

40. Koukos G, Polytarchou C, Kaplan JL, Morley-Fletcher A, Gras-Miralles B, Kokkotou $\mathrm{E}$, et al. MicroRNA-124 regulates STAT3 expression and is downregulated in colon tissues of pediatric patients with ulcerative colitis. Gastroenterology. 2013;145(4):842-52.

41. Pekow JR, Dougherty U, Mustafi R, Zhu H, Kocherginsky M, Rubin DT, et al. miR143 and miR-145 are downregulated in ulcerative colitis: putative regulators of inflammation and protooncogenes. Inflamm Bowel Dis. 2012;18(1):94-100.

42. Kim JH, Lee $\mathrm{CH}$, Lee $\mathrm{SW}$. Hepatitis $\mathrm{C}$ virus infection stimulates transforming growth factor- $\beta 1$ expression through up-regulating miR-192. J Microbiol. 2016;54(7):520-6.

43. Godwin JG, Ge X, Stephan K, Jurisch A, Tullius SG, lacomini J. Identification of a microRNA signature of renal ischemia reperfusion injury. Proc Natl Acad Sci U S A. 2010;107(32):14339-44.

44. O'Connell RM, Kahn D, Gibson WS, Round JL, Scholz RL, Chaudhuri AA, et al. MicroRNA-155 promotes autoimmune inflammation by enhancing inflammatory T cell development. Immunity. 2010;33(4):607-19. 
45. Rodriguez A, Vigorito E, Clare S, Warren MV, Couttet P, Soond DR, et al. Requirement of bic/microRNA-155 for normal immune function. Science. 2007;316(5824):608-11.

46. Polytarchou C, Hommes DW, Palumbo T, Hatziapostolou M, Koutsioumpa M, Koukos G, et al. MicroRNA214 is associated with progression of ulcerative colitis, and inhibition reduces development of colitis and colitis-associated cancer in mice. Gastroenterology. 2015;149(4):981-92.

47. Li M, Zhang S, Qiu Y, He Y, Chen B, Mao R, et al. Upregulation of miR-665 promotes apoptosis and colitis in inflammatory bowel disease by repressing the endoplasmic reticulum stress components XBP1 and ORMDL3. Cell Death Dis. 2017;8(3):e2699.

48. Miner PB Jr, Wedel MK, Xia S, Baker BF. Safety and efficacy of two dose formulations of alicaforsen enema compared with mesalazine enema for treatment of mild to moderate left-sided ulcerative colitis: a randomized, doubleblind, active-controlled trial. Aliment Pharmacol Ther. 2006;23(10):1403-13.

49. Schetter AJ, Leung SY, Sohn JJ, Zanetti KA, Bowman ED, Yanaihara N, et al. MicroRNA expression profiles associated with prognosis and therapeutic outcome in colon adenocarcinoma. JAMA. 2008;299(4):425-36.

50. Valeri N, Gasparini P, Braconi C, Paone A, Lovat F, Fabbri M, et al. MicroRNA-21 induces resistance to 5 -fluorouracil by down-regulating human DNA MutS homolog 2 (hMSH2). Proc Natl Acad Sci U S A. 2010;107(49):21098-103.

51. Deng J, Lei W, Fu JC, Zhang L, Li JH, Xiong JP. Targeting miR-21 enhances the sensitivity of human colon cancer HT-29 cells to chemoradiotherapy in vitro. Biochem Biophys Res Commun. 2014;443(3):789-95.

52. Sun D, Yu F, Ma Y, Zhao R, Chen X, Zhu J, et al. MicroRNA-31 activates the RAS pathway and functions as an oncogenic MicroRNA in human colorectal cancer by repressing RAS p21 GTPase activating protein 1 (RASA1). J Biol Chem. 2013:288(13):9508-18.

53. Sun D, Wang C, Long S, Ma Y, Guo Y, Huang Z, et al. C/EBP-ß-activated microRNA-223 promotes tumour growth through targeting RASA1 in human colorectal cancer. Br J Cancer. 2015;112(9):1491-500.

54. Wang CJ, Stratmann J, Zhou ZG, Sun XF. Suppression of microRNA-31 increases sensitivity to 5 -FU at an early stage, and affects cell migration and invasion in HCT-116 colon cancer cells. BMC Cancer. 2010;10:616.

55. Borralho PM, Kren BT, Castro RE, da Silva IB, Steer CJ, Rodrigues CM. MicroRNA-143 reduces viability and increases sensitivity to 5-fluorouracil in HCT116 human colorectal cancer cells. FEBS J. 2009;276(22):6689-700.

56. Zhang J, Guo H, Zhang H, Wang H, Qian G, Fan X, et al. Putative tumor suppressor miR-145 inhibits colon cancer cell growth by targeting oncogene friend leukemia virus integration 1 gene. Cancer. 2011;117(1):86-95.

57. Sachdeva M, Mo YY. MicroRNA-145 suppresses cell invasion and metastasis by directly targeting mucin 1. Cancer Res. 2010;70(1):378-87.

58. Zhang J, Sun Q, Zhang Z, Ge S, Han ZG, Chen WT. Loss of microRNA-143/ 145 disturbs cellular growth and apoptosis of human epithelial cancers by impairing the MDM2-p53 feedback loop. Oncogene. 2013;32(1):61-9.

59. Yan $X$, Chen $X$, Liang $H$, Deng $T$, Chen W, Zhang S, et al. miR-143 and miR145 synergistically regulate ERBB3 to suppress cell proliferation and invasion in breast cancer. Mol Cancer. 2014;13:220.

60. Liu M, Lang N, Qiu M, Xu F, Li Q, Tang Q, et al. miR-137 targets Cdc42 expression, induces cell cycle G1 arrest and inhibits invasion in colorectal cancer cells. Int J Cancer. 2011;128(6):1269-79.

61. Mudduluru G, Ceppi P, Kumarswamy R, Scagliotti GV, Papotti M, Allgayer H. Regulation of Axl receptor tyrosine kinase expression by miR-34a and miR199a/b in solid cancer. Oncogene. 2011;30(25):2888-99.

62. Hansen TF, Christensen RD, Andersen RF, Sørensen FB, Johnsson A, Jakobsen A. MicroRNA-126 and epidermal growth factor-like domain 7-an angiogenic couple of importance in metastatic colorectal cancer. Results from the Nordic ACT trial. Br J Cancer. 2013;109(5):1243-51.

63. Zhou Y, Feng $X$, Liu YL, Ye SC, Wang H, Tan WK, et al. Down-regulation of miR-126 is associated with colorectal cancer cells proliferation, migration and invasion by targeting IRS-1 via the AKT and ERK1/2 signaling pathways. PLoS One. 2013;8(11):e81203.

64. Akao Y, Noguchi S, lio A, Kojima K, Takagi T, Naoe T. Dysregulation of microRNA-34a expression causes drug-resistance to 5-FU in human colon cancer DLD-1 cells. Cancer Lett. 2011;300(2):197-204.

65. Li J, Chen Y, Zhao J, Kong F, Zhang Y. miR-203 reverses chemoresistance in p53-mutated colon cancer cells through downregulation of Akt2 expression. Cancer Lett. 2011;304(1):52-9.

66. Zhou Y, Wan G, Spizzo R, Ivan C, Mathur R, Hu X, et al. miR-203 induces oxaliplatin resistance in colorectal cancer cells by negatively regulating ATM kinase. Mol Oncol. 2014;8(1):83-92.
67. Fesler A, Zhai H, Ju J. miR-129 as a novel therapeutic target and biomarker in gastrointestinal cancer. Onco Targets Ther. 2014;7:1481-5.

68. Karaayvaz M, Zhai H, Ju J. miR-129 promotes apoptosis and enhances chemosensitivity to 5-fluorouracil in colorectal cancer. Cell Death Dis. 2013:4:e659.

69. Wang F, Zhang P, Ma Y, Yang J, Moyer MP, Shi C, et al. NIRF is frequently upregulated in colorectal cancer and its oncogenicity can be suppressed by let-7a microRNA. Cancer Lett. 2012;314(2):223-31.

70. Zhai H, Song B, Xu X, Zhu W, Ju J. Inhibition of autophagy and tumor growth in colon cancer by miR-502. Oncogene. 2013;32(12):1570-9.

71. Shi C, Yang Y, Xia Y, Okugawa Y, Yang J, Liang Y, et al. Novel evidence for an oncogenic role of microRNA-21 in colitis-associated colorectal cancer. Gut. 2016;65(9):1470-81.

72. Benderska N, Dittrich AL, Knaup S, Rau TT, Neufert C, Wach S, et al. miRNA26b overexpression in ulcerative colitis-associated carcinogenesis. Inflamm Bowel Dis. 2015;21(9):2039-51.

73. Rokavec M, Öner MG, Li H, Jackstadt R, Jiang L, Lodygin D, et al. J Clin Invest. 2014;124(4):1853-67.

74. He C, Yu T, Shi Y, Ma C, Yang W, Fang L, et al. Gastroenterology. 2017; 152(6):1434-48.

75. Ma J, Yang Y, Fu Y, Guo F, Zhang X, Xiao S, et al. PIAS3-mediated feedback loops promote chronic colitis-associated malignant transformation. Theranostics. 2018;8(11):3022-37.

76. Schwarz DS, Hutvágner G, Du T, Xu Z, Aronin N, Zamore PD. Asymmetry in the assembly of the RNAi enzyme complex. Cell. 2003;115(2):199-208.

77. Schwarz DS, Hutvágner G, Haley B, Zamore PD. Evidence that siRNAs function as guides, not primers, in the Drosophila and human RNAi pathways. Mol Cell. 2002;10(3):537-48.

78. Nathwani AC, Tuddenham EG, Rangarajan S, Rosales C, Mclntosh J, Linch DC, et al. Adenovirus-associated virus vector-mediated gene transfer in hemophilia B. N Engl J Med. 2011;365(25):2357-65.

79. Lindsay JO, Ciesielski CJ, Scheinin T, Brennan FM, Hodgson HJ. Local delivery of adenoviral vectors encoding murine interleukin 10 induces colonic interleukin 10 production and is therapeutic for murine colitis. Gut. 2003;52(7):981-7.

80. Lindsay J, Van Montfrans C, Brennan F, Van Deventer S, Drillenburg P, Hodgson $\mathrm{H}$, et al. IL-10 gene therapy prevents TNBS-induced colitis. Gene Ther. 2002:9(24):1715-21.

81. Mingozzi F, Maus MV, Hui DJ, Sabatino DE, Murphy SL, Rasko JE, et al. CD8(+) T-cell responses to adeno-associated virus capsid in humans. Nat Med. 2007;13(4):419-22.

82. Zhang Y, Wang Z, Gemeinhart RA. Progress in microRNA delivery. J Control Release. 2013;172(3):962-74.

83. Zhang Y, Cristofaro P, Silbermann R, Pusch O, Boden D, Konkin T, et al. Engineering mucosal RNA interference in vivo. Mol Ther. 2006;14(3):336-42.

84. Zuo L, Huang Z, Dong L, Xu L, Zhu Y, Zeng K, et al. Targeting delivery of anti-TNFalpha oligonucleotide into activated colonic macrophages protects against experimental colitis. Gut. 2010;59(4):470-9.

85. Huang Z, Ma J, Chen M, Jiang H, Fu Y, Gan J, et al. Dual TNF-a/IL-12p40 interference as a strategy to protect against colitis based on miR-16 precursors with macrophage targeting vectors. Mol Ther. 2015;23(10):1611-21.

86. Huang Z, Gan J, Jia L, Guo G, Wang C, Zang Y, et al. An orally administrated nucleotide-delivery vehicle targeting colonic macrophages for the treatment of inflammatory bowel disease. Biomaterials. 2015;48:26-36.

87. Dai $X$, Chen $X$, Chen $Q$, Shi L, Liang $H$, Zhou Z, et al. MicroRNA-193a-3p reduces intestinal inflammation in response to microbiota via Downregulation of colonic PepT1. J Biol Chem. 2015;290(26):16099-115.

88. Zhang M, Viennois E, Prasad M, Zhang Y, Wang L, Zhang Z, et al. Edible ginger-derived nanoparticles: a novel therapeutic approach for the prevention and treatment of inflammatory bowel disease and colitisassociated cancer. Biomaterials. 2016;101:321-40.

89. Alvarez-Erviti L, Seow Y, Yin H, Betts C, Lakhal S, Wood MJ. Delivery of siRNA to the mouse brain by systemic injection of targeted exosomes. Nat Biotechnol. 2011;29(4):341-5.

90. Ohno S, Takanashi M, Sudo K, Ueda S, Ishikawa A, Matsuyama N, et al. Systemically injected exosomes targeted to EGFR deliver antitumor microRNA to breast cancer cells. Mol Ther. 2013;21(1):185-91.

91. Yin $Y$, Cai $X$, Chen $X$, Liang $H$, Zhang $Y$, Li J, et al. Tumor-secreted miR-214 induces regulatory $T$ cells: a major link between immune evasion and tumor growth. Cell Res. 2014;24(10):1164-80.

92. Zhang P, Wang C, Zhao J, Xiao A, Shen Q, Li L, et al. Near infrared-guided smart Nanocarriers for MicroRNA-controlled release of doxorubicin/siRNA with intracellular ATP as fuel. ACS Nano. 2016;10(3):3637-47. 
93. Liang G, Zhu Y, Jing A, Wang J, Hu F, Feng W, et al. Cationic microRNAdelivering nanocarriers for efficient treatment of colon carcinoma in xenograft model. Gene Ther. 2016;23(12):829-38.

94. Sureban SM, May R, Mondalek FG, Qu D, Ponnurangam S, Pantazis $\mathrm{P}$, et al. Nanoparticle-based delivery of siDCAMKL-1 increases microRNA-144 and inhibits colorectal cancer tumor growth via a Notch-1 dependent mechanism. J Nanobiotechnology. 2011;9:40.

95. Zheng B, Chen L, Pan CC, Wang JZ, Lu GR, Yang SX, et al. Targeted delivery of miRNA-204-5p by PEGylated polymer nanoparticles for colon cancer therapy. Nanomedicine (Lond). 2018;13(7):769-85.

96. Dai L, Wang W, Zhang S, Jiang Q, Wang R, Dai L, et al. Vector-based miR-15a/16-1 plasmid inhibits colon cancer growth in vivo. Cell Biol Int. 2012;36(8):765-70

97. Li Y, Duo Y, Zhai P, He L, Zhong K, Zhang Y, et al. Dual targeting delivery of miR-328 by functionalized mesoporous silica nanoparticles for colorectal cancer therapy. Nanomedicine (Lond). 2018. https://doi.org/10.2217/nnm2017-0353.

98. Li Y, Duo Y, Bi J, Zeng X, Mei L, Bao S, et al. Targeted delivery of anti-miR155 by functionalized mesoporous silica nanoparticles for colorectal cancer therapy. Int J Nanomedicine. 2018;13:1241-56.

99. Ling $H$, Fabbri $M$, Calin GA. MicroRNAs and other non-coding RNAs as targets for anticancer drug development. Nat Rev Drug Discov. 2013;12(11): 847-65.

100. de Pontual L, Yao E, Callier P, Faivre L, Drouin V, Cariou S, et al. Germline deletion of the miR-17 92 cluster causes skeletal and growth defects in humans. Nat Genet. 2011;43(10):1026-30.

101. Kota J, Chivukula RR, O'Donnell KA, Wentzel EA, Montgomery CL, Hwang $\mathrm{HW}$, et al. Therapeutic microRNA delivery suppresses tumorigenesis in a murine liver cancer model. Cell. 2009;137(6):1005-17.

102. Huse JT, Brennan C, Hambardzumyan D, Wee B, Pena J, Rouhanifard SH, et al. The PTEN-regulating microRNA miR-26a is amplified in high-grade glioma and facilitates gliomagenesis in vivo. Genes Dev. 2009;23(11):1327-37.

103. Kent OA, McCall MN, Cornish TC, Halushka MK. Lessons from miR-143/145: the importance of cell-type localization of miRNAs. Nucleic Acids Res. 2014;42(12):7528-38.

104. Davis S, Propp S, Freier SM, Jones LE, Serra MJ, Kinberger G, et al. Potent inhibition of microRNA in vivo without degradation. Nucleic Acids Res. 2009;37(1):70-7.

105. Kim DH, Rossi JJ. Strategies for silencing human disease using RNA interference. Nat Rev Genet. 2007:8(3):173-84.

106. Pecot CV, Calin GA, Coleman RL, Lopez-Berestein G, Sood AK. RNA interference in the clinic: challenges and future directions. Nat Rev Cancer. 2011;11(1):59-67.

107. Gamazon ER, Ziliak D, Im HK, LaCroix B, Park DS, Cox NJ, et al. Genetic architecture of microRNA expression: implications for the transcriptome and complex traits. Am J Hum Genet. 2012;90(6):1046-63.

108. Whitehead KA, Langer R, Anderson DG. Knocking down barriers: advances in siRNA delivery. Nat Rev Drug Discov. 2009;8(2):129-38.

109. Pfeffer SR, Yang CH, Pfeffer LM. The role of miR-21 in Cancer. Drug Dev Res. 2015;76(6):270-7.

110. Ferraro A, Kontos CK, Boni T, Bantounas I, Siakouli D, Kosmidou V, et al. Epigenetic regulation of miR-21 in colorectal cancer: ITGB4 as a novel miR21 target and a three-gene network (miR-21-ITGB4-PDCD4) as predictor of metastatic tumor potential. Epigenetics. 2014;9(1):129-41.

111. Wei C, Li L, Kim IK, Sun P, Gupta S. NF-KB mediated miR-21 regulation in cardiomyocytes apoptosis under oxidative stress. Free Radic Res. 2014;48(3):282-91.

112. McDaniel DK, Eden K, Ringel VM, Allen IC. Emerging roles for noncanonical NF-KB signaling in the modulation of inflammatory bowel disease pathobiology. Inflamm Bowel Dis. 2016;22(9):2265-79.

113. Bouchie A. First microRNA mimic enters clinic. Nat Biotechnol. 2013;31(7):577.

114. Janssen HL, Reesink HW, Lawitz EJ, Zeuzem S, Rodriguez-Torres M, Patel K, et al. Treatment of HCV infection by targeting microRNA. N Engl J Med. 2013;368(18):1685-94.

115. Elmén J, Lindow M, Silahtaroglu A, Bak M, Christensen M, Lind-Thomsen A, et al. Antagonism of microRNA-122 in mice by systemically administered LNA-antimiR leads to up-regulation of a large set of predicted target mRNAs in the liver. Nucleic Acids Res. 2008;36(4):1153-62 Epub 2007.

116. Elmén J, Lindow $M$, Schütz $S$, Lawrence $M$, Petri $A$, Obad $S$, et al. LNAmediated microRNA silencing in non-human primates. Nature. 2008; 452(7189):896-9.
117. Ottosen S, Parsley TB, Yang L, Zeh $K$, van Doorn $L$, van der Veer $E$, et al. In vitro antiviral activity and preclinical and clinical resistance profile of miravirsen, a novel anti-hepatitis $C$ virus therapeutic targeting the human factor miR-122. Antimicrob Agents Chemother. 2015;59(1):599-608.

118. Cortez MA, Valdecanas D, Zhang X, Zhan Y, Bhardwaj V, Calin GA, et al. Therapeutic delivery of miR-200c enhances radiosensitivity in lung cancer. Mol Ther. 2014;22(8):1494-503.

119. Beg MS, Brenner AJ, Sachdev J, Borad M, Kang YK, Stoudemire J, et al. Phase I study of MRX34, a liposomal miR-34a mimic, administered twice weekly in patients with advanced solid tumors. Investig New Drugs. 2017;35(2):180-8.

120. Zandwijk NV, Pavlakis N, Kao S, Clarke S, Lee A, Brahmbhatt H, et al. P1. 02MesomiR 1: A Phase I study of TargomiRs in patients with refractory malignant pleural mesothelioma (MPM) and lung cancer (NSCLC). Annals of Oncology, 2015(suppl 2):ii16.

121. Trajkovski M, Hausser J, Soutschek J, Bhat B, Akin A, Zavolan M, et al. MicroRNAs 103 and 107 regulate insulin sensitivity. Nature. 2011;474(7353):649-53.

122. Gomez IG, MacKenna DA, Johnson BG, Kaimal V, Roach AM, Ren S, et al. Anti-microRNA-21 oligonucleotides prevent Alport nephropathy progression by stimulating metabolic pathways. J Clin Invest. 2015;125(1):141-56.

\section{Ready to submit your research? Choose BMC and benefit from:}

- fast, convenient online submission

- thorough peer review by experienced researchers in your field

- rapid publication on acceptance

- support for research data, including large and complex data types

- gold Open Access which fosters wider collaboration and increased citations

- maximum visibility for your research: over 100M website views per year

At BMC, research is always in progress.

Learn more biomedcentral.com/submissions 